\title{
Higher difference structure of some discrete processes
}

\author{
AY Shahverdian ${ }^{1,2^{*}}$, A Kilicman $^{3}$ and RB Benosman ${ }^{4}$
}

"Correspondence: svrdn@yerphi.am ${ }^{1}$ Institute for Informatics and Automation Problems, National Academy of Sciences of Armenia, Yerevan, Armenia ${ }^{2}$ Department of Applied Mathematics, State Engineering University of Armenia, Terian St. 105, Yerevan, 0009, Armenia

Full list of author information is available at the end of the article

\begin{abstract}
A method for analyzing discrete processes based on consideration of their higher difference structure is presented. Two kinds of processes, deterministic systems (discrete dynamical systems) and the sequences of random binary independent variables, are considered. Two main statements, computation of higher absolute differences and restoring the original by a given higher order difference image, are studied. Some applications to dynamical systems are given.
\end{abstract}

MSC: 39B12; 39A50; 37E05

Keywords: higher differences; discrete dynamical systems; independent random processes

\section{Introduction}

This paper presents some results on difference analysis, which is a method for studying irregular time series and orbits of discrete dynamical systems. Such an analysis, suggested in [1-3], is based on consideration of higher absolute differences taken from successive terms of a given time series or orbits of a given system. This is motivated by an observation that some natural systems (e.g., the visual cortex, [4]) process the information contained in signals difference structure. The difference analysis reveals some new aspects of nonlinearity; for instance, a difference analog for Lyapunov exponent [5], used for chaos discrimination, is introduced [3].

The content of this paper is as follows. In Section 2 deterministic processes are considered, Section 3 deals with random independent binary processes. In both cases, we are interested in computation of higher order absolute differences, recovering the original by a given difference image as well as in the limiting behavior of difference processes. Section 4 contains the proofs of results presented.

\section{Deterministic processes}

This section considers higher absolute differences taken from successive terms of discrete deterministic systems. Two main problems, computation of higher differences and restoring the original, are studied. Some applications to discrete dynamical systems are given.

\subsection{Higher absolute differences of deterministic processes}

In difference analysis, a given time series or an orbit $X$ is decomposed into two constituents: the sign component $\mathrm{S}$ and the magnitude (or height) component $\mathrm{H}$. The $\mathrm{S}$-component reflects the alternation in monotony (increase/decrease) of higher order 
absolute differences taken from successive terms of $X$. The component $\mathrm{S}$ does not depend on the very exact values of these differences, while the component $\mathrm{H}$ consists of these absolute differences and does not depend on their sign distribution. At every step (associated with the order of differences) of the decomposition process, the orbit $X$ can be completely restored by first terms of $X$ and the two components. Since the behavior of $X$ at infinity is determined by the limiting behavior of its $\mathrm{S}$ - and $\mathrm{H}$-components, such an analysis can also be useful in dynamical systems (see, e.g., [3]).

Let us present some formal definitions. Let $X$ be some infinite numerical sequence

$$
X=\left(f_{1}, f_{2}, \ldots, f_{n}, \ldots\right) .
$$

This can be some time series, experimental data, or an orbit of iterates of some onedimensional map. We consider the consecutive absolute differences,

$$
H_{n}^{(0)}=f_{n}, \quad H_{n}^{(m+1)}=\left|H_{n+1}^{(m)}-H_{n}^{(m)}\right| \quad(m \geq 0, n \geq 1),
$$

define ( $m$ th order) difference sequence, $H^{(m)}\left(=H_{1}^{(m)}\right)$, and consider binary sequences

$$
S_{m}=\left(\delta_{1}^{(m)}, \delta_{2}^{(m)}, \ldots, \delta_{n}^{(m)}, \ldots\right), \quad \delta_{n}^{(m)}= \begin{cases}+1, & H_{n+1}^{(m)} \geq H_{n}^{(m)}, \\ -1, & H_{n+1}^{(m)}<H_{n}^{(m)} .\end{cases}
$$

In this way, a given time series $X$ is decomposed into two other, the sign and magnitude components $\left(S_{m}\right)_{m=1}^{\infty}$ and $\left(H^{(m)}\right)_{m=1}^{\infty}$. We call $S_{m}$ and $H^{(m)}$ the $m$ th S- and H-components of $X$, respectively, and denote $S_{m}=S_{m}(X), H^{(m)}=H^{(m)}(X)$, and $H_{m}(X)=\left(H_{n}^{(m)}\right)_{n=1}^{\infty}$.

In this paper, we are mostly interested in behavior of H-components. Equation (2) is the basic one for studying the $\mathrm{H}$-component: due to this equation, for most cases, any property of series $H_{1}^{(m)}\left(=H^{(m)}\right)$ implies the same property of every series $H_{n}^{(m)}$ with $n \geq 2$. For instance, it follows that if for some $n \geq 1$ (for some $m \geq 1$ ) the limit

$$
H_{n}^{(\infty)}=\lim _{m \rightarrow+\infty} H_{n}^{(m)} \quad\left(H_{\infty}^{(m)}=\lim _{n \rightarrow+\infty} H_{n}^{(m)}\right)
$$

exists, then for every $n^{\prime} \geq n$ (for every $m^{\prime}>m$ ) the limit $H_{n^{\prime}}^{(\infty)}\left(H_{\infty}^{\left(m^{\prime}\right)}\right)$ also exists.

The following theorem asserts that every absolute difference $H_{n}^{(m)}(X)$ is a linear form of the original $X$ and presents a formula for its computation. The rule (5) below is a generalization of the well-known additive scheme for constructing the Pascal triangle of binomial coefficients. This theorem is a consequence of relations (2); the proof (conducted by induction) is straightforward and is omitted.

Theorem 2.1 For every time series (1) the $H_{n}^{(m)}$ can be computed by the formula

$$
H_{n}^{(m)}=k_{m, n}^{(0)} f_{n}+k_{m, n}^{(1)} f_{n+1}+\cdots+k_{m, n}^{(m)} f_{n+m},
$$

where the coefficients $k_{m, n}^{(i)} \in \mathbb{Z}$ are constructed recurrently: for $p \geq 1$

$$
k_{1, n}^{(0)}=-k_{1, n}^{(1)}=-\delta_{n}^{(0)}, \quad k_{p+1, n}^{(i)}= \begin{cases}-\delta_{n}^{(p)} k_{p, n}^{(i)}, & i=0, \\ \delta_{n}^{(p)}\left(k_{p, n+1}^{(i-1)}-k_{p, n}^{(i)}\right), & 1 \leq i \leq p, \\ \delta_{n}^{(p)} k_{p, n+1}^{(i-1)}, & i=p+1 .\end{cases}
$$




\subsection{Restoring the original by difference image}

At every $m$ th step (associated with the order $m$ of differences) of the decomposition process, the original time series can be completely restored by its first $m$ entries and the two components $\mathrm{S}$ and $\mathrm{H}$. Indeed, the following theorem provides us with analytical expressions for computation of the original $X$ by the components $S_{1}, S_{2}, \ldots, S_{m}, H^{(m)}$ and first $m$ terms $f_{1}, \ldots f_{m}$ (below, it is denoted $\left.\left[f_{1}, \ldots, f_{k}\right]=H^{(k)}\right)$ :

Theorem 2.2 Let $m \geq 1$ be given, $H=\left(h_{n}\right)_{n=1}^{\infty}$ be some infinite numerical sequence, $h_{n} \geq 0$ and $S_{k}=\left(s_{k, n}\right)_{n=1}^{\infty}, s_{k, n}= \pm 1,0 \leq k \leq m$ be some infinite binary sequences. Let $X=\left(f_{n}\right)_{n=1}^{\infty}$ be defined as follows: $f_{1}, \ldots, f_{m}$ are arbitrary and for every natural $n \geq 1$

$$
f_{n}=f_{1}+\sum_{k=2}^{m}\left[f_{1}, \ldots, f_{k}\right] \sum_{i=1}^{n-k+1} B_{n, i, k-2}+\sum_{i=1}^{n-m} h_{i} B_{n, i, m-1},
$$

where $\sum_{i}^{j}=0$ if $j<i$ and $B_{n, i, p} \in \mathbb{Z}$ are constructed recurrently: for $p \geq 1$

$$
B_{n, i, 0}=s_{0, i}, \quad B_{n, i, p}=s_{p, i} \sum_{j=i+1}^{n-p} B_{n, j, p-1} .
$$

Then for all $1 \leq n \leq m$ the relations

$$
S_{n}(X)=S_{n} \quad \text { and } \quad H_{m}(X)=H
$$

hold.

We note that Eq. (7) represents a countable infinite system of higher order difference equations (which are nonlinear since we deal with absolute differences) and its solution by Eq. (6) is given. The proof of this theorem is straightforward (by induction) and is omitted.

\subsection{Application to dynamical systems}

We consider the orbits of the maps $f$ defined on the unit segment $I=[0,1]$ and the limiting (as the order $m$ of differences tends to $+\infty$ ) behavior of their difference series $H^{(m)}$. We claim that this behavior is mainly due to some function $\mathcal{H}\left(=\mathcal{H}_{f}\right)$ which can be determined as a solution to some difference-functional equation.

Let $f: I \rightarrow I$ be a map defined on the numerical segment $I$ and $X$ be an orbit,

$$
X=\left(f^{(0)}(x), f^{(1)}(x), \ldots, f^{(n)}(x), \ldots\right)
$$

that is, we put in (1) $f_{n}=f^{(n)}(x)$, where $f^{(n)}\left(f^{(0)}(x) \equiv x, f^{(n+1)}=f \circ f^{(n)}\right), n \geq 0$ denote the iterates of the map $f$. Theorem 2.1 yields that $H^{(m)}$ is some 'iterate polynomial',

$$
H^{(m)}(x)=\sum_{n=0}^{m} k_{m}^{(n)} f^{(n)}(x)
$$

where $k_{m}^{(n)} \in \mathbb{Z}$ and $k_{m}^{(0)}+\cdots+k_{m}^{(m)}=0$. Let $\mathcal{D}_{f}$ denote the collection of such $x \in I$, for each of which $H^{(m)}(x)$ is convergent as $m \rightarrow \infty$. Therefore, one can consider the following 
limiting function $\mathcal{H}\left(=\mathcal{H}_{f}\right)$ defined on $\mathcal{D}_{f}$ :

$$
\mathcal{H}(x)=\lim _{m \rightarrow \infty} H^{(m)}(x)
$$

$\mathcal{H}$, being defined through the magnitude orbit $H^{(m)}$, reflects also the behavior at infinity of every $n$th series $\left(H_{n}^{(m)}\right)_{m=1}^{\infty}$. Indeed, it follows from (2) that, for $x \in \mathcal{D}_{f}$ and every $n \geq 1, f^{(n)}(x)$ is also found in $\mathcal{D}_{f}\left(\right.$ i.e., $f^{(n)}\left(\mathcal{D}_{f}\right) \subseteq \mathcal{D}_{f}$ for all $\left.n \geq 1\right)$. Then since $H_{n}^{(m)}(x)=$ $H_{1}^{(m)}\left(f^{(n)}(x)\right)$, it follows that

$$
\lim _{m \rightarrow \infty} H_{n}^{(m)}(x)=\mathcal{H}\left(f^{(n)}(x)\right)
$$

The next statement presents a difference equation for computation of the limiting function $\mathcal{H}$ and establishes the special property of such functions: once vanished, $\mathcal{H}$ remains zero on all the further orbit of the map $f$. In the following two statements, $[a]$ denotes the entire part of number $a$ (maximal integer which does not exceed $a$ ) and $\{a\}=a-[a]$ is its fractional part.

Theorem 2.3 For functions $\mathcal{H}$ defined by Eq. (9), the following statements are true:

(1) The function $\mathcal{H}$ satisfies the following functional equation:

$$
|\mathcal{H}(x)-\mathcal{H}(f(x))|=\mathcal{H}(x) .
$$

(2) If for some $x \in \mathcal{D}_{f}$ we have $\mathcal{H}(x)=0$, then $\mathcal{H}\left(f^{(n)}(x)\right)=0$ for all $n \geq 0$.

(3) If for some $x \in \mathcal{D}_{f}$ we have $\mathcal{H}(x) \neq 0$, then there is a number $N, 0 \leq N \leq \log [1 / \mathcal{H}(x)]$ such that

$$
\mathcal{H}\left(f^{(n)}(x)\right)= \begin{cases}2^{n} \mathcal{H}(x), & 0 \leq n \leq N \\ 0, & n>N\end{cases}
$$

The proof of this theorem is straightforward: the point (1) of theorem is a consequence of Eq. (2) and points (2) and (3) follow directly from the point (1).

Particularly, this theorem immediately implies the following.

Corollary 2.4 Let, for some $x \in I$, the semi-orbit

$$
\ldots, f^{(-3)}(x), f^{(-2)}(x), f^{(-1)}(x), f(x)
$$

be infinite. Then $\mathcal{H}\left(f^{(n)}(x)\right)=0$ for all large enough $n \in \mathbb{Z}$.

For instance, if $f$ is such that $f(I)=I$ (e.g., this can be the so-called 4-logistic map $f(x)=$ $4 x(1-x)$ or Bernoulli shift $f(x)=\{2 x\}$ studied in deterministic chaos $[5,6])$ and $\mathcal{D}_{f}=I$, then for every $x \in I$ the semi-orbit (11) is infinite and hence, by Corollary 2.4, for such $f, \mathcal{H}$ is identically zero on every periodic orbit: if $x \in \operatorname{Per}(f)$, then $\mathcal{H}\left(f^{n}(x)\right)=0$ for every $n \in \mathbb{Z}$. Particularly, it follows that if $f: I \rightarrow I$ is such that $f(I)=I$ and $\operatorname{Per}(f)$ is dense on $I$, then the null-set $\left\{x \in I: \mathcal{H}_{f}(x)=0\right\}$ is also dense on $I$. 


\section{Random independent discrete processes}

This section considers the higher absolute differences taken from successive terms of random independent binary sequences (see also [7]). The same two problems as in Section 2, computation of higher order absolute differences and restoring the original, are studied. When proving Theorem 3.2, we underline some connections of difference analysis with discrete models of self-organized criticality (SOC), a physics-originated theory (e.g., $[8,9])$ which studies the large systems of interacting microsystems.

\subsection{Higher absolute differences of random binary processes}

Let $\xi$ be a discrete binary random independent process

$$
\xi=\left(\xi_{1}, \xi_{2}, \ldots, \xi_{n}, \ldots\right)
$$

(the variables $\xi_{n}$ are independent and take binary values 0 and 1 with some positive probabilities). The absolute differences

$$
\xi_{n}^{(0)}=\xi_{n}, \quad \xi_{n}^{(k+1)}=\left|\xi_{n+1}^{(k)}-\xi_{n}^{(k)}\right| \quad(k \geq 0, n \geq 1)
$$

also take binary values with some positive probabilities, and hence one can consider the random difference processes $\xi^{(k)}=\left(\xi_{1}^{(k)}, \xi_{2}^{(k)}, \ldots, \xi_{n}^{(k)}, \ldots\right)$ assuming their independence. For a binary variable $\xi$, we assign the distribution of probabilities in the form $P(\xi=\lambda)=\frac{1}{2}(1+$ $\left.(-1)^{\lambda} \pi\right)$, where $\lambda \in\{0,1\}$ and $\pi \in(0,1)$. We denote $\pi=\pi(\xi)$ and characterize a variable $\xi$ by the corresponding number $\pi(\xi)$. We notice that since $\pi(\xi)=\pi(1-\xi), \pi(\xi)$ determines a variable $\xi$ uniquely only when $\xi$ is equidistributed, i.e., $\pi(\xi)=0$. In the following, we consider the binary version $\mathbb{P}$ of the Pascal triangle of binomial coefficients, $\mathbb{P}=\left\langle\alpha_{i, k}\right\rangle_{i, k}$ $(0 \leq i \leq k ; k \geq 1)$, where every $k$ th line $(k \geq 1)$ of $\mathbb{P}$ is defined by relations

$$
\alpha_{0, k}=\alpha_{k, k}=1 \quad \text { and } \quad \alpha_{i, k}=\left|\alpha_{i-1, k-1}-\alpha_{i, k-1}\right| \quad \text { for } 1 \leq i \leq k-1 .
$$

The following Theorem 3.1 is an analog for Theorem 2.1. To demonstrate the analogy, instead of $\pi(\xi)$, we use $\varphi(\xi)=-\ln \pi(\xi)$ and denote $\varphi_{n}=\varphi\left(\xi_{n}\right)$ and $\varphi_{n}^{(k)}=\varphi\left(\xi_{n}^{(k)}\right)$ $\left(=-\ln \pi\left(\xi_{n}^{(k)}\right)\right)$.

Theorem 3.1 For every process (12), $\varphi_{n}^{(k)}$ can be computed by the formula

$$
\varphi_{n}^{(k)}=\alpha_{0, k} \varphi_{n}+\alpha_{1, k} \varphi_{n+1}+\cdots+\alpha_{k, k} \varphi_{n+k}
$$

where the coefficients $\alpha_{i, k}$ are the terms of $k$ th line of the triangle $\mathbb{P}$.

\subsection{Restoring the original by difference image}

The following problem is studied: given natural $k \geq 1$ and a random sequence $\boldsymbol{\eta}=\left(\eta_{n}\right)_{n=1}^{\infty}$ to determine a random process $\xi=\left(\xi_{n}\right)_{n=1}^{\infty}$ for which the relation $\xi^{(k)}=\eta$ holds. We give a solution assuming that processes $\xi$ and $\xi^{(i)}, 1 \leq i \leq k$ are independent. Based on Theorem 3.1 and defining random sequences $\left(\xi_{n}\right)_{n=1}^{\infty}$ by their distributions $\left(\varphi_{n}\right)_{n=1}^{\infty}$, our task is reduced to the following: given $k \geq 1$ and a numerical sequence $\left(\beta_{n}\right)_{n=1}^{\infty}, \beta_{n}>0$ to determine the positive numbers $\left(\varphi_{n}\right)_{n=1}^{\infty}$ for which the relations

$$
\alpha_{0, k} \varphi_{n}+\alpha_{1, k} \varphi_{n+1}+\cdots+\alpha_{k, k} \varphi_{n+k}=\beta_{n}
$$


for $n=1,2, \ldots$ hold. The solution to this task is given by Theorem 3.2 which is an analog for Theorem 2.2. The proof of Theorem 3.2 is conducted by involving some physics-related notions such as particles, energy, etc. Particularly, this allows us to demonstrate some connections of the difference analysis with a modification of some SOC-related models.

Let $q=q(k)$ denote the number of nonzero $\alpha_{i, k}$ on $k$ th line of the triangle $\mathbb{P}$. We assume that such $\alpha_{i, k}$ are numbered in increasing order of the index $i: \alpha_{s_{i}, k}=1$, where $0=s_{0}<s_{1}<$ $\cdots<s_{q}=k$ and let us denote $h_{i}=k+1-s_{i}$. Thus, a number $q=q(k)$ and two ordered samples,

$$
\mathbf{s}=\left(s_{0}, s_{1}, \ldots, s_{q}\right), \quad \mathbf{h}=\left(h_{0}, h_{1}, \ldots, h_{q}\right),
$$

correspond to a given $k$. Equation (14) means that our task is reduced to solving some countable system of linear algebraic equations; since, for every $i$ which is not contained in the sample $\mathbf{s}$ from Eq. (15), we have $\alpha_{i, k}=0$, the system of equations (14) coincides with the system (16) below.

In the next formulation, for a positive number $a,[a]$ denotes the entire part of $a$ (maximal integer which does not exceed $a$ ), and for natural $a$ and $b$, we denote $\{a / b\}=\left\{\begin{array}{ll}r, & r \neq 0 \\ b, & r=0\end{array}\right.$, where $r$ is residual from dividing $a$ by $b$ (and $r=a$ if $a<b$ ).

Theorem 3.2 Let $k \geq 2$ be given, $q=q(k)$, and $\mathbf{s}=\left(s_{i}\right)_{i=0}^{q}$ and $\mathbf{h}=\left(h_{i}\right)_{i=0}^{q}$ be defined by Eq. (15). Let us consider the system of linear algebraic equations

$$
\varphi_{n+s_{0}}+\varphi_{n+s_{1}}+\cdots+\varphi_{n+s_{q}}=\beta_{n}
$$

(where $n=1,2, \ldots$ and $\beta_{n}$ are free terms) and define the numbers $\varphi_{n}$ as follows:

(1) $\varphi_{1}, \varphi_{2}, \ldots, \varphi_{k}$ are positive and arbitrary, and for $1 \leq T \leq k-s_{q-1}, \varphi_{k+T}$ is equal to

$$
\varphi_{k+T}=\beta_{T}-\sum_{i=1}^{q-1} \varphi_{T+s_{i-1}} ;
$$

(2) for $T \geq k-s_{q-1}+1, \varphi_{k+T}$ is equal to

$$
\varphi_{k+T}=\sum_{m=1}^{T}(-1)^{m} \beta_{1+\left[\frac{T-1}{m}\right]}+\sum_{i=1}^{q-1} \sum_{s=1}^{q-1} \sum_{n=1}^{\left[T / h_{s}\right]}(-1)^{\left[\frac{T-1-n h_{s}}{h_{i}}\right]} \varphi_{1+\left\{\frac{T-1-n h_{s}}{h_{i}}\right\}}
$$

Then, for every $n \in \mathbb{N}$, the sample $\left(\varphi_{n+s_{0}}, \varphi_{n+s_{1}}, \ldots, \varphi_{n+s_{q}}\right)$ satisfies Eq. (16).

It can be noticed from formulation of this theorem that the sought $\varphi_{m}$ (whose index $m$ is given as $\left.m=n+s_{i}\right)$ are such that $\varphi_{1}, \varphi_{2}, \ldots, \varphi_{k}$ are positive and arbitrary, while for $m \geq$ $k+1$, we have $\varphi_{m}=\varphi_{m}\left(\beta_{1}, \beta_{2}, \ldots, \beta_{m} ; \varphi_{1}, \varphi_{2}, \ldots, \varphi_{k}\right)$; that is, the above-mentioned restored process $\xi$ is not unique but depends on $k$ arbitrary positive numerical parameters.

To prove Theorem 3.2, we use the following physics-related model, which can be treated as a modification of some lattice models (e.g., the BTW-model, [9]) of SOC. Let us describe the model that we use. Let, for $k \geq 2, \mathbb{L}_{k}=[1, k] \times[1, k]$ be a lattice from $\mathbb{N}^{2}$. At every moment of discrete time $t=1,2, \ldots$ at the vertices of $\mathbb{L}_{k}$, which are found above the diagonal 
$L$ connecting the vertices $(1,1)$ and $(k, k)$, some material particles with positive or negative mass (or charge) are situated. Each particle performs uniform rectilinear motion with the unit speed on the vertical segments $L_{i}$ connecting the vertices $\left(s_{i}, s_{i}\right)$ and $\left(s_{i}, k\right)(1 \leq i \leq q)$ of $\mathbb{L}_{k}$. At the (initial) moment $t=1$, all the particles are found on $L$ and possess the unit mass. Each moment $t$, when a number $(=p)$ of particles reach simultaneously the upper side $\{(k, i): 1 \leq i \leq k\}$ of the lattice (one can see that such particles should possess the same mass $m$ ), these particles disappear, and at the next moment $t+1$, at every position on $L$ a new particle of the mass - pm emerges (and the motion of particles continues). In SOC terminology, the $p$ particles hit the threshold (the upper side of the square $\mathbb{L}_{k}$ ), and an avalanche of intensity $p$ at the moment $t$ in the system $S$ of the moving particles occurs.

We consider a function (a potential) $U=U(y)$ defined on the upper triangle $\{(x, y) \in$ $\mathbb{L}_{k}: x \leq y$ \} which depends only on the coordinate $y, 1 \leq y \leq k$. Our task related to the above-presented model is to compute the potential energy

$$
E(t)=\sum_{i, s} m_{i, s}(t) U\left(y_{i, s}(t)\right)
$$

of the system $S$ of particles on $\mathbb{L}_{k}$ for every moment $t$; here, $y_{i, s}$ and $m_{i, s}$ denote the $y$-coordinate and mass of the particle which at the moment $t$ is found at the vertex $(i, s) \in \mathbb{L}_{k}$. The proof of Theorem 3.2 is based on the following lemma.

Lemma 3.3 For every $t=1,2, \ldots$, the total (potential) energy of the system $S$ can be computed by the formula

$$
E(t)=-\sum_{i=1}^{q-1} \sum_{s=1}^{q-1} \sum_{n=1}^{\left[t / h_{s}\right]}(-1)^{\left[\frac{t-1-n h_{s}}{h_{i}}\right]} U\left(1+\left\{\frac{t-1-n h_{s}}{h_{i}}\right\}\right) .
$$

\section{Proof of results}

Proof of Theorem 3.1 Let $m \geq 2, \mathbf{x}=\left(x_{1}, x_{2}, \ldots, x_{m}\right) \in \mathbb{R}^{m}$, and we consider the polynomials

$$
P_{m}(\mathbf{x})=\left(1+x_{1}\right)\left(1+x_{2}\right) \cdots\left(1+x_{m}\right) \quad\left(=\sum x_{1}^{\varepsilon_{1}} x_{2}^{\varepsilon_{2}} \cdots x_{m}^{\varepsilon_{m}}\right),
$$

where summation is extended over all binary samples $\left(\varepsilon_{1}, \ldots, \varepsilon_{m}\right)$ of length $m$. If for $\lambda \in$ $\{0,1\}$ one considers the polynomials (the sum of binary variables is understood as the sum by $\bmod (2))$

$$
P_{m}^{(\lambda)}(\mathbf{x})=\sum_{\varepsilon_{1}+\cdots+\varepsilon_{m}=\lambda} x_{1}^{\varepsilon_{1}} x_{2}^{\varepsilon_{2}} \cdots x_{m}^{\varepsilon_{m}}
$$

then denoting $-\mathbf{x}=\left(-x_{1}, \ldots,-x_{m}\right)$ it follows that for $\lambda \in\{0,1\}$,

$$
P_{m}^{(\lambda)}(\mathbf{x})=\frac{1}{2}\left[P_{m}(\mathbf{x})+(-1)^{\lambda} P_{m}(-\mathbf{x})\right] .
$$


Indeed, we have $P_{m}^{(\lambda)}(-\mathbf{x})=(-1)^{\lambda} P_{m}^{(\lambda)}(\mathbf{x})$, which yields Eq. (21). This implies that, for $\lambda \in$ $\{0,1\}$ and $\mathbf{x} \in \mathbb{R}^{m}$, the identity

$$
\sum_{\varepsilon_{1}+\cdots+\varepsilon_{m}=\lambda} x_{1}^{\varepsilon_{1}} x_{2}^{\varepsilon_{2}} \cdots x_{m}^{\varepsilon_{m}}=\frac{1}{2}\left(1+(-1)^{\lambda} \frac{1-x_{1}}{1+x_{1}} \frac{1-x_{2}}{1+x_{2}} \cdots \frac{1-x_{m}}{1+x_{m}}\right) \prod_{i=0}^{m}\left(1+x_{i}\right)
$$

(it is assumed that $x_{i} \neq-1$ ) holds.

On the other hand, one can prove (we omit the proof which can be conducted by the induction method) that for $k \geq 1$ and $\lambda \in\{0,1\}$,

$$
\left(\xi_{n}^{(k)}=\lambda\right)=\bigcup_{\left\langle\varepsilon_{0}, \ldots, \varepsilon_{k}\right\rangle=\lambda}\left(\xi_{n+k}=\varepsilon_{k}, \xi_{n+k-1}=\varepsilon_{k-1}, \ldots, \xi_{n}=\varepsilon_{0}\right)
$$

where it is denoted $\left\langle\varepsilon_{0}, \ldots, \varepsilon_{k}\right\rangle=\sum_{i=0}^{k} \varepsilon_{i} \alpha_{i, k} \bmod (2)$. Then the independence of the processes $\xi^{i}$ that we assumed implies

$$
P\left(\xi_{n}^{(k)}=\lambda\right)=\sum_{\left\langle\varepsilon_{0}, \ldots, \varepsilon_{k}\right\rangle=\lambda} P\left(\xi_{n}=\varepsilon_{0}\right) P\left(\xi_{n+1}=\varepsilon_{1}\right) \cdots P\left(\xi_{n+k}=\varepsilon_{k}\right) .
$$

Denoting $p_{n}=P\left(\xi_{n}=0\right), \bar{p}_{n}=P\left(\xi_{n}=1\right)\left(p_{n}+\bar{p}_{n}=1\right)$ and $\delta_{i}=\varepsilon_{s_{i}}$ (see Eq. (15)), one obtains

$$
\begin{aligned}
P\left(\xi_{n}^{(k)}=\lambda\right) & =\sum_{\delta_{0}+\cdots+\delta_{q}=\lambda} p_{n+s_{0}}^{1-\delta_{0}} \bar{p}_{n+s_{0}}^{\delta_{0}} \cdots p_{n+s_{q}}^{1-\delta_{q}} \bar{p}_{n+s_{q}}^{\delta_{q}} \\
& =\bar{p}_{n+s_{0}} \bar{p}_{n+s_{1}} \cdots \bar{p}_{n+s_{q}} \sum_{\delta_{0}+\cdots+\delta_{q}=\lambda}\left(\frac{p_{n+s_{0}}}{\bar{p}_{n+s_{0}}}\right)^{\delta_{0}}\left(\frac{p_{n+s_{1}}}{\bar{p}_{n+s_{1}}}\right)^{\delta_{1}} \cdots\left(\frac{p_{n+s_{q}}}{\bar{p}_{n+s_{q}}}\right)^{\delta_{q}} .
\end{aligned}
$$

Now, by applying Eq. (22), one obtains Eq. (13). Theorem 3.1 is proved.

Proof of Lemma 3.3 For the case of a single particle with the (initial) mass equal to -1 moving on a vertical segment $L_{i}$ connecting the vertices $\left(s_{i}, s_{i}\right)$ and $\left(s_{i}, k\right)(1 \leq i \leq q)$ of $\mathbb{L}_{k}$, one can compute that

$$
U(t)=1+\left\{\frac{t-1}{h_{i}}\right\}, \quad m(t)=(-1)^{1+\left[\frac{t-1}{h_{i}}\right]} .
$$

That is, for every $t$, the energy of a single particle moving on $L_{i}$ is equal to

$$
E_{i}(t)=(-1)^{1+\left[\frac{t-1}{h_{i}}\right]} U\left(1+\left\{\frac{t-1}{h_{i}}\right\}\right) .
$$

Considering the case of many particles, we note that on any segment $L_{i}$ a new particle in the system at a moment $t$ emerges if and only if $t$ is of the form $t=n h_{s}+1$ (for some $n \geq 1$ and some $1 \leq s \leq q-1)$. For particles that emerged as a result of attainment of the threshold during the motion on $L_{i}$, the coordinate and mass are computed by (23); for particles that emerged on $L_{i}$ as a result of attainment of the threshold during the motion on some $L_{s}, s \neq i$, the coordinate and mass are computed by the same formula (23) but in 
a 'shifted' time: $t \rightarrow t-n h_{s}$. Therefore, for the total energy $E^{(i)}$ of the particles situated on a given vertical segment $L_{i}$, one obtains

$$
E^{(i)}(t)=E_{i}(t)+\sum_{s=1}^{q-1} \sum_{n=1}^{\left[t / h_{s}\right]} E_{i}\left(t-n h_{s}\right) .
$$

Hence, for every $t$, the total energy of all the particles in the system $S$ is equal to

$$
E(t)=\sum_{i=1}^{q-1} \sum_{s=1}^{q-1} \sum_{n=1}^{\left[t / h_{s}\right]} E_{i}\left(t-n h_{s}\right),
$$

which due to Eq. (24) coincides with Eq. (20). Lemma 3.3 is proved.

Proof of Theorem 3.2 Since $s_{q}=k$, Eq. (16) can be re-written as

$$
\varphi_{k+n}=\beta_{n}-\varphi_{n+s_{1}}-\varphi_{n+s_{2}}-\cdots-\varphi_{n+s_{q-1}} \quad(n \geq 1) .
$$

Let us denote $p=k-s_{q-1}$. One can see that $1 \leq p \leq k-1$ and $\varphi_{p+s_{q-1}}=\varphi_{k}$ and then Eq. (26) can be re-written as follows:

$$
\begin{aligned}
& \varphi_{k+1}=\beta_{1}-\varphi_{1+s_{1}}-\varphi_{1+s_{2}}-\cdots-\varphi_{1+s_{q-1}}, \\
& \varphi_{k+2}=\beta_{2}-\varphi_{2+s_{1}}-\varphi_{2+s_{2}}-\cdots-\varphi_{2+s_{q-1}}, \\
& \cdots \\
& \varphi_{k+p}=\beta_{p}-\varphi_{p+s_{1}}-\varphi_{p+s_{2}}-\cdots-\varphi_{k}
\end{aligned}
$$

and (for $T \geq 1$ )

$$
\begin{aligned}
& \varphi_{k+p+1}=\beta_{p+1}-\varphi_{p+1+s_{1}}-\varphi_{p+1+s_{2}}-\cdots-\varphi_{k+1}, \\
& \varphi_{k+p+2}=\beta_{p+2}-\varphi_{p+1+s_{1}}-\varphi_{p+1+s_{2}}-\cdots-\varphi_{k+2}, \\
& \cdots \\
& \varphi_{k+p+T}=\beta_{p+T}-\varphi_{p+1+s_{1}}-\varphi_{p+1+s_{2}}-\cdots-\varphi_{k+T},
\end{aligned}
$$

One can see that (27) coincides with the point (1) of the theorem, and this point of the theorem is proved.

We base the proof of the point (2) of the theorem on the above-defined lattice model and use the Gauss substitution method for solving linear systems. With each variable of the form $\varphi_{n+s_{m}}$, where $1 \leq n \leq p$ and $1 \leq m \leq q-1$ ( $c f$. Eq. (27)), we associate a particle which is found on $m$ th vertical segment $L_{m}$ of length $h_{m}$ in the upper triangle of $\mathbb{L}_{k}$ (and if $m$ is fixed, the index $n$ enumerates a particle's position on this segment). Equations (27) and (28) and the substitution method assign the following dynamics of index $p+1+s_{m}$ (and the corresponding particles) with respect to variable $p$ : if this index does not exceed $k$ (that is, the corresponding particle is found in the lattice $\mathbb{L}_{k}$ ) then $p$ increases, and if in some equation from the system (28) it appears that for some $p$ and $m$ the inequality 
$p+1+s_{m} \geq k+1$ holds, then the variable $\varphi_{p+1+s_{m}}$ in this equation is replaced (according to the corresponding equation in (27)) by the sum

$$
\beta_{s-p}-\varphi_{s-p+s_{1}}-\varphi_{s-p+s_{2}}-\cdots-\varphi_{s-p+s_{q-1}} \quad\left(=\varphi_{k+(s-p)}\right)
$$

In terms of the lattice model, the inequality $p+1+s_{m} \geq k+1$ is interpreted as the threshold attainment and the substitution of $\varphi_{p+1+s_{m}}$ is interpreted as an event of emergence of $q-1$ new particles on each of $q-1$ positions of the diagonal $L$. One can see that if this variable substitution procedure is sequently applied to all the equations in the system (28), we obtain the above-defined lattice model of moving particles. Then, by applying Lemma 3.3 (where the time variable $t$ coincides with the variable $T$ in Eq. (28)) and assuming that the potential $U$ (Section 3.2) is assigned as $U(y)=\varphi_{1+y}$, we obtain the sought Eq. (18). Theorem 3.2 is proved.

\section{Competing interests}

The authors declare that they have no competing interests.

\section{Authors' contributions}

AYS participated in preparing the most part of the paper and the most part of statements and proofs. AK participated in the proof of Theorem 3.2, in the statements on random processes and drafted the manuscript. RBB participated in the statement of Theorem 2.3, proof of Lemma 3.3, preparing the text on SOC model, and drafted the manuscript. All authors read and approved the final manuscript.

\section{Author details}

${ }^{1}$ Institute for Informatics and Automation Problems, National Academy of Sciences of Armenia, Yerevan, Armenia. ${ }^{2}$ Department of Applied Mathematics, State Engineering University of Armenia, Terian St. 105, Yerevan, 0009, Armenia. ${ }^{3}$ Department of Mathematics and Institute for Mathematical Research, University Putra Malaysia, Serdang, Selangor 43400 UPM, Malaysia. ${ }^{4}$ Vision and Natural Computation Group, Vision Institute, University Pierre et Marie Curie-Paris 6, 17 rue Moreau, Paris, 75012, France.

\section{Acknowledgements}

Authors thank the editing group for corrections in the linguistics of the manuscript.

Received: 20 October 2012 Accepted: 8 November 2012 Published: 26 November 2012

\section{References}

1. Shahverdian, AY, Apkarian, AV: On irregular behavior of neuron spike trains. Fractals 7, 93-103 (1999)

2. Shahverdian, AY: A finite-difference method for analyzing one-dimensional nonlinear systems. Fractals $8,49-65$ (2000)

3. Shahverdian, AY, Apkarian, AV: A difference characteristic for one-dimensional nonlinear systems. Commun. Nonlinear Sci. Numer. Simul. 12, 233-242 (2007)

4. Miller, KD: In: Domany, E, van Hemmen, JL, Schulten, K (eds.) Models of Neural Networks, vol. 3. Springer, New York (1996)

5. Schuster, HG: Deterministic Chaos. Physik-Verlag, Weinheim (1984)

6. Sharkovsky, AN, Maisterenko, YL, Romanenko, EY: Difference Equations and Applications. Nauka, Kiev (1986)

7. Shahverdian, AY: Minimal Lie algebra, fine limits, and dynamical systems. Reports of Armenian Natl. Acad. Sci. 112, 160-169 (2012)

8. Shahverdian, AY, Apkarian, AV: Avalanches in networks of weakly coupled phase-shifting rotators. Commun. Math Sci. 6, 217-234 (2008)

9. Bak, P, Tang, C, Wiesenfeld, K: Self-organized criticality: an explanation of 1/f noise. Phys. Rev. Lett. 59, 381-384 (1987)

doi:10.1186/1687-1847-2012-202

Cite this article as: Shahverdian et al.: Higher difference structure of some discrete processes. Advances in Difference Equations 2012 2012.202. 